\section{Training of Spanish Allergology Residents in Pediatric Allergology: A Survey of the Current Situation}

Ruiz Hornillos $\mathrm{J}^{1}$, Nevot Falcó $\mathrm{S}^{1}$, Olaguibel JM², Ibáñez $\mathrm{MD}^{1}$

${ }^{1}$ Teaching Interest Group of the Child Allergy Committee of the Spanish Society of Allergology and Clinical Immunology, Spain ${ }^{2}$ National National Commission of the Specialty of Allergology, Spain

J Investig Allergol Clin Immunol 2017; Vol. 27(3): 203 doi: 10.18176/jiaci.0153

Key words: Allergology. Pediatric allergy. Training. Specialty.

Palabras clave: Alergología. Alergia pediátrica. Formación. Especialidad.

\section{To the Editor:}

Despite the recent repeal of the common trunk training plan by the Spanish Supreme Court, we must consider what improvements can be made in the training of allergologists in the near future. This challenge is even more relevant, given that the European Union seeks to standardize training programs for specialists, including allergologists, by the year 2020 and thus facilitate the free movement of specialists within the European Union.

One of the main objectives of the Pediatric Allergy Committee of the Spanish Society of Allergology is to promote the training of residents in pediatric allergology.

In order to properly plan the current and future needs of specialists in allergology, it is necessary to know where we are today. The official training program for allergologists, which appears in the Spanish Official State Bulletin (BOE 3081/2006 dated 20 September), recommends a 5-month rotation in pediatrics, preferably during the first 2 years of the specialty, although it does not establish other specific stages. Consequently, the Teaching Working Group of the SEAIC Children's Allergy Committee designed a specific survey with the objective of knowing differences in training in pediatric allergology for allergology residents in Spain.

The survey was sent to the tutors of allergology residents at the 42 allergology training units (ATUs) of Spanish hospitals certified for training. Replies were received from all 42 ATUs. Analysis of the data revealed the following results:

- Specific training in pediatric allergology is carried out in all certified ATUs and lasts 1 to 3 months in 7 units, 4-6 months in $19,7-9$ months in 6 , and $>9$ months in 10 . In $90.5 \%$ of the ATUs, this stage is during the third or fourth year of training. In $50 \%$, residents move to another ATU to receive training (71.4\% of them to an ATU in another city and $28.6 \%$ to an ATU in the same city).

- Residents received the training from allergists in their own service in 18 ATUs $(42.9 \%)$, pediatricians in 4 $(9.5 \%)$, pediatric allergists in $11(26.2 \%)$, and allergists with exclusive practice in children in $9(21.4 \%)$.

- Finally, 38 of the ATUs (90.5\%) had programmed training periods in general pediatrics (4-9 months in 24 [57.1\%] and $1-3$ months in 14 [33.3\%]) that were generally run during the first year of the residency programme. Furthermore, in 24 ATUs (57.1\%), the residents were involved in emergency pediatrics on-calls $(70 \%$ in the emergency department and $30 \%$ on general wards).

- With regard to the contents of the training program, all of the residents received formal training and acquired skills specifically in children's disease in the main speciality areas: respiratory allergy, food allergy, skin allergy, and drug allergy. They also received specific training in common diagnostic methods and procedures such as lung function testing, including bronchial challenge with methacholine in $74 \%$ of ATUs, nasal examination in $81 \%$, food immunotherapy in $93 \%$, management of eosinophilic esophagitis in $86 \%$, and food masking techniques for controlled exposure tests in $71 \%$.

We conclude that all allergology residents in Spain receive specific training in pediatric allergology, although the survey detected some deficiencies, namely, an excessively short training programme $(<6$ months $)$ and a lack of training in pediatric emergency wards in some ATUs.

The National Commission of the Specialty of Allergology is faced with both a challenge and an opportunity: the challenge of designing the new training program taking into account the importance of pediatric allergology in the professional development of allergologists, and the opportunity to further improve training in pediatric allergology and thus resolve current deficiencies.

\section{Funding}

The study was supported by the Spanish Society of Allergology and Clinical Immunology.

\section{Conflicts of Interest}

The authors declare that they have no conflicts of interest.

\section{- JM Olaguibel}

E-mail: jm.olaguibel.ribero@cfnavarra.es 